\title{
Tangential distribution of cell type and direction selectivity in monkey area MT
}

\author{
ANTONIA CINIRA M. DIOGO, BRUSS LIMA, JULIANA G.M. SOARES \& \\ RICARDO GATTASS
}

\begin{abstract}
We studied the multiunit responses to moving and static stimuli from 585 cell clusters in area MT using multi-electrode arrays. Our aim was to explore if MT columns exhibit any larger-scale tangential organization or clustering based on their response properties. Neurons showing both motion and orientation selectivity were classified into four categories: 1- Type I (orientation selectivity orthogonal to the axis of motion); 2- Type II (orientation selectivity coaxial to the axis of motion); 3- Type DS (significant response to moving stimuli, but non-significant response to static stimuli); and 4- Type OS (significant orientation selectivity, but non-significant direction selectivity). Type I (34\%), Type II (24\%) and Type DS (32\%) clusters were the most predominant and may be associated with different stages of motion processing in MT. On the other hand, the rarer Type OS (9\%) may be integrating motion and form processing. Type I and unidirectional sites were the only classes to exhibit significant clustering. Type OS sites showed a trend for clustering, which did not reach statistical significance. We also found a trend for unidirectional sites to have bidirectional sites as neighbors. In conclusion, neuronal clustering associated with these four categories may be related to distinct MT functional circuits.
\end{abstract}

Key words: cell types, direction selectivity, orientation selectivity, extrastriate cortex, visual system, primates.

\section{INTRODUCTION}

Intrinsic circuits and feedforward projections give rise to receptive field properties that are increasingly more complex as one advances along the visual hierarchy (Hubel \& Wiesel 1968, Gross et al. 1972, Gattass et al. 2005). This organization is compatible with the notion that the response properties of the neurons at different visual processing stages are related to specific attributes of perception (Barlow et al. 1967, Barlow 1986). The extrastriate cortex in primates contains multiple visual areas (Van Essen \& Zeki 1978, Gattass \& Gross 1981, Gross et al. 1981, Gattass et al. 1988, Kaas 1989,
Neuenschwander et al. 1994). The study of their receptive field properties has shown results that are compatible with both parallel (Martin \& Whitteridge 1984) and serial (Hubel \& Wiesel 1962) neuronal processing. Indeed, while the processing of the visual attributes is distributed across multiple areas (Zeki 1969, Van Essen \& Zeki 1978) and streams (Ungerleider \& Mishkin 1982), receptive field size becomes gradually larger at each successive hierarchical stage (Gattass et al. 1985).

Early work of extrastriate areas attributed color processing to a region in the pre-lunate gyrus (Zeki 1983a, b), while visual motion was processed at the superior temporal sulcus (STS) 
(Zeki 1974). Furthermore, initial investigations on the posterior portion of the STS revealed at least three distinct visual areas: the middle temporal area (MT) (Gattass \& Gross 1981, Ungerleider \& Desimone 1986, Fiorani et al. 1989) and two middle superior temporal (MST) areas (Newsome \& Wurtz 1981, Newsome et al. 1986, Desimone \& Ungerleider 1986). Analysis of their receptive field properties indicated that visual motion processing becomes more elaborate as one progresses from area MT to area MST (Zeki 1969, Albright 1984, Albright et al. 1984, Movshon et al. 1985, Rodman et al. 1989, 1990, Newsome \& Wurtz 1981, Hikosaka et al. 1988, Tanaka et al. 1986, Wurtz et al. 1990). Within this hierarchical framework we hypothesize that area MT is structured into modular domains, perhaps through the clustering of specific cell types into hypercolumns or stripes, which supports the gradual sophistication of motion computation eventually found in area MST.

MT neurons can be classified into at least four different functional cell types based on their receptive field properties: Type I, Type II, DS and OS. Type I neurons respond to components of the moving visual stimulus, Type II neurons respond to global object motion, Type DS neurons are selective to the direction of stimulus motion, and Type OS neurons respond primarily to stimulus orientation, possibly being part of a different processing circuit altogether (Movshon et al. 1985, Albright et al. 1984, Stoner \& Albright 1998). In order to investigate the hypothesis that these different cell types are part of distinct MT modules participating in distinct stages of motion processing, we investigated the distribution of these four cell types across the tangential dimension of area MT in the capuchin monkey. More specifically, we were interested in investigating if these cell types exhibited a random distribution, or if they clustered in any particular way. Using the same rational, we also looked for clustering of direction selectivity (uni-, bi- and pan-directionality) across the tangential dimension of MT. Regarding cell type, we found a predominance of Type I and Type DS cells, a result also found in the macaque. Type I was the only one of the four cell type classes to exhibit significant clustering across MT surface. Type OS cells showed a trend for clustering, but the results did not reach statistical significance. Regarding direction selectivity, unidirectional sites was the only one of the three direction selectivity classes to exhibit significant clustering. A preliminary account of this data was presented in abstract form (Diogo et al. 1999).

\section{MATERIALS AND METHODS}

\section{Animal subjects}

We recorded neuronal activity from two adult capuchin monkeys (Sapajus apella, formely named (ebus apella), each weighing between 2 and $3 \mathrm{~kg}$. Each animal was a subject in three to five recording sessions. Other analyses of the data acquired during these experiments were presented in a previous study (Diogo et al. 2003). All experimental protocols followed the National Institutes of Health guidelines for animal care and use and were approved by the Institutional Animal Care and Use Committee at Instituto de Biofísica Carlos Chagas Filho/Universidade Federal do Rio de Janeiro.

\section{Animal preparation and maintenance}

General procedures were similar to those employed by Albright et al. (1984) and Diogo et al. 2003). Briefly, a stainless-steel cylinder and a head bolt were affixed to the animal's skull with screws and dental acrylic. Surgical procedures were performed under aseptic conditions using ketamine anesthesia $(20 \mathrm{mg} / \mathrm{kg}$ ). For the recording sessions, animals were anesthetized 
initially using ketamine $(20 \mathrm{mg} / \mathrm{kg}$ ) followed by halothane $(2.0 \%)$ in a $7: 3$ mixture of nitrous oxide and oxygen. Animals were paralyzed by a continuous infusion of pancuronium bromide (0.1 $\mathrm{mg} / \mathrm{kg} / \mathrm{h}$ ) and artificially ventilated. Halothane was discontinued once paralysis became stable, and anesthesia was maintained by nitrous oxide and oxygen and by a continuous infusion of fentanyl citrate (0.003 $\mathrm{mg} / \mathrm{kg} / \mathrm{h}$ ). Body temperature was maintained at $37-38^{\circ} \mathrm{C}$ with a heating pad, and respiratory parameters were adjusted to give an end-tidal carbon dioxide level of approximately $4 \%$. We induced cycloplegia and mydriasis with topical applications of $10 \%$ and $1 \%$ solutions of phenylephrine and atropine, respectively. The contralateral eye was focused on a tangent screen at a distance of $57 \mathrm{~cm}$, and the ipsilateral eye was occluded. Recording sessions generally continued for 10-18 h.

\section{Microelectrode arrays for electrophysiological imaging}

We used linear microelectrode arrays to sample neuronal response properties at nodes within a $2 \mathrm{D}$ rectilinear matrix. The arrays were constructed by gluing together a set of microelectrode guide tubes, such that the electrode tips formed points on a line or nearly so (estimated error, $\pm 30 \mu \mathrm{m})$. Each array was advanced parallel to the
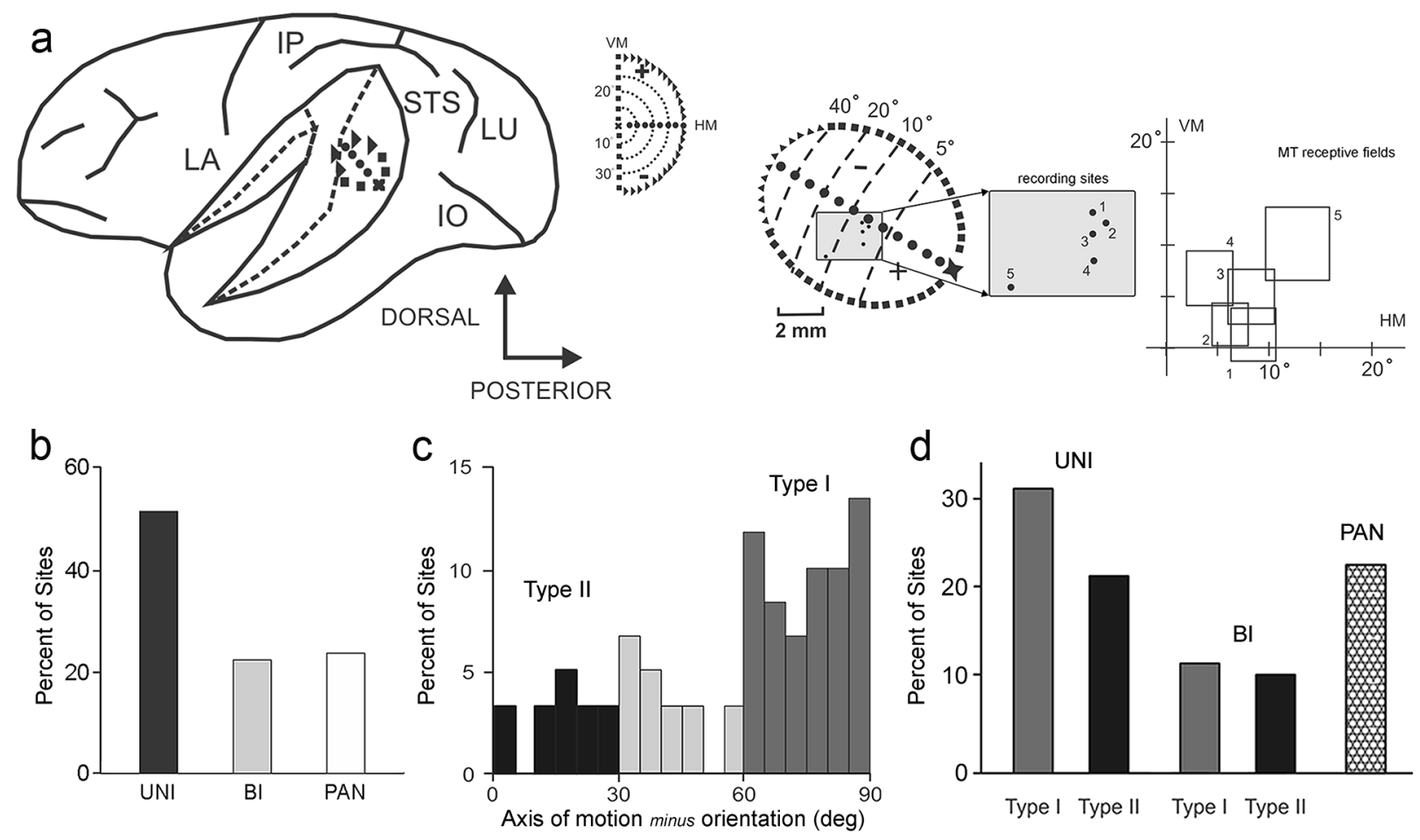

Figure 1. Placement of the planar electrode array and the distribution of neuronal response properties in area MT. (a) Left: Schematic representation of the left hemisphere of a capuchin monkey's brain. The lateral and superior temporal sulci are shown open in order to evidence MT's location and visual topography (see inset). Right: Five example recording sites in MT alongside their corresponding receptive fields. (b) Distribution of directional selectivity across three classes of sites: unidirectional (UNI), bidirectional (BI) and pan-directional (PAN). (c) Distribution of Type I and Type II sites. In order to compute the coaxiality of orientation vs. direction selectivity, we computed the difference between the preferred axis of motion and preferred orientation. (d) Distribution of cell types across 5 categories: Type I and Type II (each split for uni- and bidirectional selective sites), and PAN selective neurons. Abbreviations: IP, intraparietal sulcus; LA, lateral sulcus; STS, superior temporal sulcus; LU, lunate sulcus; IO, inferior occipital sulcus; VM, vertical meridian; HM, horizontal meridian. 
cortical surface in $200 \mu \mathrm{m}$ steps, and neuronal responses were recorded from each electrode, so the outcome of this procedure was a 2D matrix of samples. The spatial resolution of the matrix was $200 \times 350 \mu \mathrm{m}$ (see Figure 1 in Diogo et al. 2003).

\section{Electrophysiological recording strategies}

Recordings were confined to the cortical region representing the lower quadrant of the contralateral visual field, within $15^{\circ}$ of the fovea. The microelectrode array entered the brain dorsal-caudally at an angle of $12^{\circ}$ posterior to the frontal plane. In both monkeys, the penetrations were approximately tangential (within $10^{\circ}-12^{\circ}$ ) to the laminar boundaries of area MT. Multi-unit activity comprising an estimated one to three neurons in each recording site were isolated using an amplitude discriminator. The resulting events were digitized and stored using the CORTEX program (courtesy of the National Institutes of Health, NIH-USA).

\section{Visual stimulation}

The computer-generated stimuli used were of two types: asymmetric square-wave gratings (1.1 cycle $/{ }^{\circ}$, duty cycle $=0.33$ ) and random-dot arrays (dot size $=0.5^{\circ}$, dot density $=0.45 \mathrm{dot} /$ $\mathrm{deg}^{2}$ ). Direction selectivity was studied using stimuli moving at a speed of $3^{\circ}$ per second (random dots and gratings moved in one of 12 directions, steps of $30^{\circ}$ between directions), while orientation selectivity was investigated using flashed stationary gratings with the same physical characteristics as the moving gratings. In addition, we used the stationary flashed random-dot arrays to measure the responsiveness of the site to a static stimulus without orientation. The stimulus was presented on a 21 -inch raster-scan video monitor (frame rate $=60 \mathrm{~Hz}$ ) and viewed through a $25^{\circ}$ diameter circular aperture. The resulting full-field stimulus covered the entire region comprising the simultaneously recorded receptive fields. Each stimulus (type and direction of motion) was presented for 10 trials on a pseudo-random schedule. Each trial was comprised of three epochs: 1- 200 ms blank period; 2- stimulus presented stationary during 400 ms; 3- the same stimulus moving continuously in a single direction of motion during 1000 ms. Gratings and dots were presented in separate blocks of trials.

Orientation selectivity was studied using stationary gratings ( 6 orientations, $30^{\circ}$ steps). Each grating orientation was presented for 10 trials on a pseudo-random schedule. Each trial was comprised of three epochs: 1- 200 ms blank period; 2- stimulus presented stationary during 400 ms; 3- 400 ms blank period.

During a pilot experiment, we noticed that our full-field square gratings could potentially induce stimulus after-effects. We were concerned that this effect might be carried over to the next trial and interfere with the neuronal activity to the new condition being presented. Indeed, analysis of 66 recording sites during a pilot experiment for which we used a 1000 ms intertrial interval revealed that the neuronal response to a certain direction of motion interfered with the neuronal activity of the subsequent trial. A 2000 ms intertrial period abolished this effect and was thereby the intertrial period we adopted for all the data presented here.

\section{Statistical analysis}

We quantified the stimulus-driven neuronal activity by computing the mean firing rate during the stimulus presentation period. Spontaneous neuronal activity was computed by averaging the periods with no visual stimulation. Visual responsive sites were detected by comparing the spontaneous vs. stimulation periods using a paired t-test. Sites where $p>0.05$ 
were categorized as non-responsive. Direction selectivity was tested using analysis of variance (ANOVA) across the conditions comprising the different directions of visual motion. Sites where $p>0.05$ were categorized as pan-directional. Sites where neuronal responses to one direction of motion was significantly greater than the responses to the other directions were classified as unidirectional. For these cases, the response to the opposite direction was often null or inhibited relative to spontaneous activity. If two opposing directions elicited responses that were significantly greater than the rest, the site was classified as bi-directional. For some bidirectional sites, there was a response inhibition to the movement orthogonal to the axis of motion. Additionally, we compared direction and orientation selectivity across different stimulation conditions (i.e., gratings vs. dots).

We quantified the neuronal stimulus selectivity for a given recording site by fitting either one Gaussian function to the activity elicit by static gratings (i.e., analysis of orientation selectivity) or two Gaussian functions to the activity elicited by moving gratings or moving dots (i.e., analysis of direction selectivity). The parameters obtained by the Gaussian fit were used for subsequent analysis (see Diogo et al. 2003 for more details).

Neurons were classified into four categories relative to cell type:1-Typel:orientation selectivity orthogonal to the axis of preferred motion; 2Type II: orientation selectivity coaxial to the axis of preferred motion; 3- Type DS: significant response to moving stimuli, but no response to static stimuli; 4- Type OS: pan-directional (no direction selectivity), but selective to stimulus orientation. Note that Type I, Type II and DS cells could be either unidirectional or bidirectional neurons, since both of the latter classifications satisfied the criterium for direction selectivity. Finally, not all pan-directional neurons were
Type OS cells, since the latter required selectivity for orientation, while the former did not.

\section{Correlation between the cell type and direction selectivity}

Each recording site received two classifications: 1- regarding cell type: Type I, II, DS or OS; 2regarding direction selectivity: unidirectional, bi-directional or pan-directional. We computed the correlation between cell type and direction selectivity using the $x^{2}$ test, which evaluated if direction selectivity was equally distributed within each cell type group. Additionally, we studied the tangential distributions of cell type and direction selectivity independently in order to test if the corresponding distributions were haphazard or if there was any evidence of clustering. Note that the two dimensions of the map have different spatial resolutions (200 x $350 \mu \mathrm{m}$ ), which correspond to the two sampling axes. Nevertheless, we were still able to compute the next neighbor distribution for cell type and direction selectivity. A random distribution (null hypothesis) would predict a $25 \%$ probability of having each one of the four different cell types (Type I, Type II, Type DS or Type OS) as neighbor. Accordingly, a random distribution would predict a $33 \%$ probability of having each one of the three categories of direction selectivity (unidirectional, bidirectional or pan-directional) as neighbor. Note that our predictions for a random distribution could change depending on the frequency of occurrence in our data sample of each cell type and each direction selectivity. The prediction used to compute the $x^{2}$ test was accordingly corrected.

\section{Cluster size and distribution}

We evaluated the distribution cell type and direction selectivity using progressive sample consensus (PROSAC, see below), next neighbor probability and 2D-Fast Fourier Transformation 
(FFT) in order to estimate the size of the modules. Analysis was applied to the entire population and to each class of sites in order to evaluate the cluster arrangement based on partially ordered scalograms and on histograms of vector magnitude for each interaction among similar sites (Z1-Zn distances).

\section{Progressive Sample Consensus (PROSAC)}

We used a randomized data mining method, named Progressive Sample Consensus (PROSAC) in order to detect clusters of spatially overlapping images (Chum \& Matas 2010). The core of the method relies on the MinHash algorithm in order to detect pairs of images with spatial overlap, the so-called cluster seed. The seeds are used as visual queries to obtain clusters that are formed as transitive closures of sets of partially overlapping images that include the seed. The PROSAC algorithm exploits the linear ordering defined on the set of correspondence by a similarity function used in establishing tentative correspondence. PROSAC samples are drawn from progressively larger sets of top-ranked correspondence. The power of the method is demonstrated on wide baseline matching problems.

For the derived size of the sampled set of correspondences as a function of the number of samples already drawn, PROSAC converges (in the worst case) towards RANSAC (Chum \& Matas 2010). RANSAC treats all correspondences equally and draws random samples uniformly from the full set.

The ordering is defined, at least implicitly, in all commonly used local matching methods, because the set of tentative correspondences is obtained by first evaluating a real-valued similarity function (or "quality") $q$ that is used as threshold to obtain the $\mathrm{N}$ correspondences. Thus, PROSAC is a robust estimator of cluster distance. The PROSAC algorithm exploits the ordering structure of the set of tentative correspondences, assuming that the ordering by similarity computed on local descriptors is better than random. The sampling on progressively larger subsets consisting of topranked correspondences brings very significant computational savings. PROSAC removes one parameter of the matching process - the threshold on the similarity function for selection of tentative correspondences. Thus, robustness against either having too few correspondences or a large number of tentative correspondences with high outlier percentage is achieved. The next neighbor probability was estimated based on the percentage of similar neighbors for each site, at different distances, in a circle of $500 \mu \mathrm{m}$ radius. Within a given cluster, the probability remains constant in a circle of approximately the size of the cluster. The dimension of the clusters was compared with the distance of halfwidth at half-height of the first (fundamental) component of the 2D-FFT power spectrum. Therefore, the size of the cluster was estimated based on both the cutoff of the first component, as well as by the distance at which the next neighbor probability falls abruptly.

\section{Histology}

At the end of a 3-5 week recording period, each animal was anesthetized with an overdose of sodium pentobarbital, and perfused through the heart with saline followed by formalin solution. The brain was removed from the skull and sectioned ( $40 \mu \mathrm{m}$ thickness) at an angle $20^{\circ}$ posterior to the frontal plane. Alternate sections were stained either for myelin or for cell bodies using the Gallyas' (1979) or the Nissl method, respectively. MT boundaries were determined based on its characteristic myeloarchitecture (Fiorani et al. 1989, Gattass \& Gross 1981, Ungerleider \& Mishkin 1979, Van Essen et al. 1981). Oblique frontal sections were 
used for the reconstruction of multi-electrode array penetrations through the cerebral cortex. These penetrations progressed along an oblique dorsal-ventral trajectory (angled $12^{\circ}$ posteriorly from the frontal plane) through the brain. Linear discontinuities and gliosis present in subsequent sections were marked in order to reconstruct, at the appropriate angle, the locations of the penetrations. Microelectrode tracks were reconstructed based on two criteria: 1- the positions of electrolytic lesions, which were made at specific points along each penetration (generally at the end), and 2stereotaxic coordinates of the penetration. Data analysis was restricted to the recording sites located in area MT, as defined by the heavily myelinated region along the floor and the lower bank of the superior temporal sulcus (STS) (see Fiorani et al. 1989).

\section{RESULTS}

We used multiple electrodes arranged in a planar array to study the motion response properties of MT sites (i.e., the multiunit neuronal activity) in two capuchin monkeys. Figure 1a illustrates the location and visuotopic organization of area MT. Also illustrated are five recording sites and their corresponding receptive fields (data from four of these sites are shown in Figures 2 and 3). Single penetrations like the ones shown had the goal of providing a coarse visuotopic map of MT, and thereby provide guideline coordinates to subsequently insert the planar electrode array.

We sampled the 2D organization of MT at three distinct planes tangential to the cortical surface. We worked with the assumption that the few nearby neurons recorded at a single electrode tip had very similar response properties, prompting them to be classified as a homogeneous 'cell type', or a site with a homogeneous selectivity to stimulus motion. In total, we studied 585 sites in area MT. Our aim was to investigate if the distribution of cell types in MT (i.e., Type I, II, DS and OS) was random or showed a significant degree of clustering. Likewise, we also investigated if the distribution of motion selectivity (i.e., unidirectional, bidirectional and pan-directional) was random or clustered. Type I and Type II cells, respectively, were defined as having their orientation selectivity axis either orthogonal or coincident with their preferred axis of motion. Type DS cells had significant direction selectivity, but no orientation selectivity. Type I, Type II and DS cells could be either unidirectional or bidirectional neurons. Type os cells were orientation selective but exhibited no direction selectivity (i.e., they were either pan-directional or exhibit no response to motion at all). In all cases, orientation selectivity was measured using static gratings.

The data were organized into two independent domains corresponding to cell type and direction selectivity. Figure 4 illustrates the distribution of cell type and direction selectivity for the tangential plane in which we obtained the largest number of responsive sites.

We found $79 \%$ of the sites to be responsive to moving gratings, and $84 \%$ of the sites to be responsive to moving dots. Eighty-five percent (85\%) of the responsive sites exhibited directional selectivity for gratings and/or dots. The remaining 15\% were pan-directional. Overall, Type I unidirectional sites were slightly more frequent (34\%) than Type DS cells (32\%), followed by Type II cells (24\%) and the least frequent Type OS cells (9\%).

\section{Cell Classes in area MT}

Figure $1 \mathrm{~b}$ shows the distribution of unidirectional, bidirectional and pan-directional sites across our entire population. These data were used for constructing the distribution map for motion selectivity. Note that the majority of 

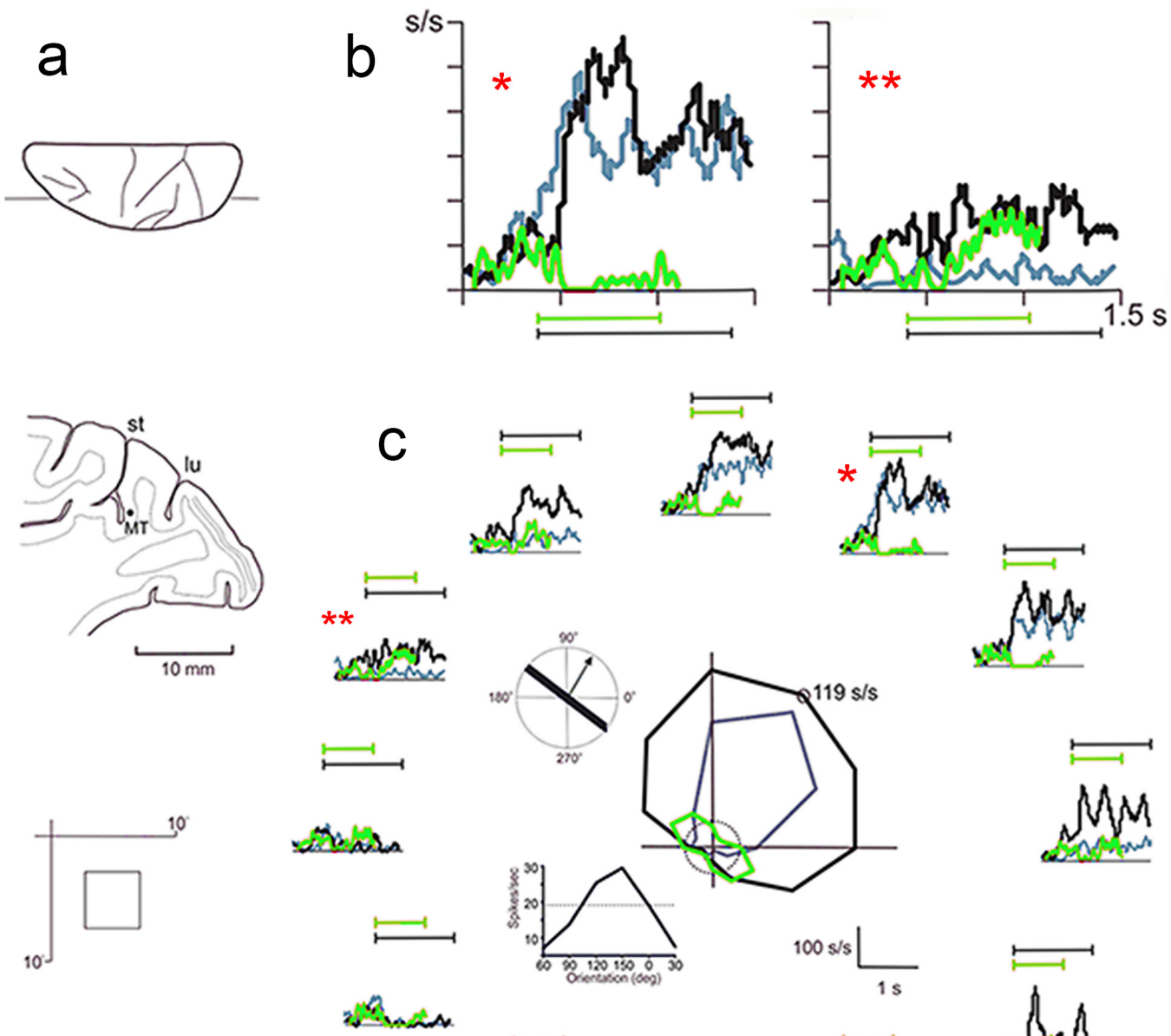

Type I Unidirectional
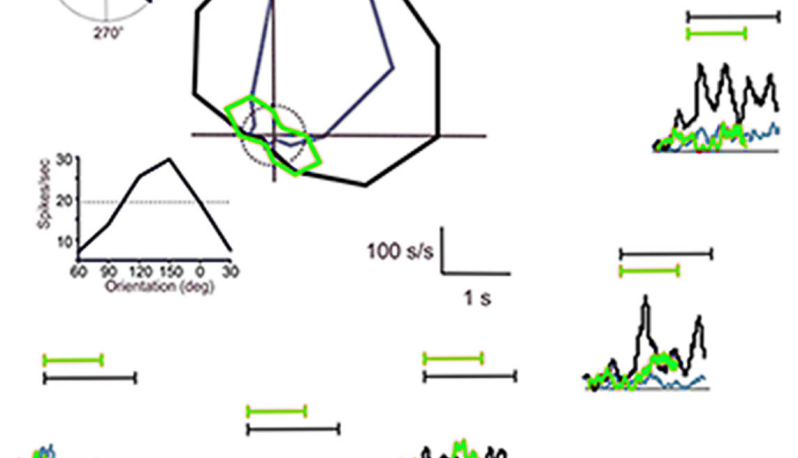

Lundur

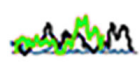

Figure 2. Example of an MT site showing Type I unidirectional activity. (a) top: Dorsal view of the brain indicating the sagittal plane illustrated in the middle panel. The black dot indicates the location of the recording site. Bottom: Receptive field of the recorded site (located within $5^{\circ}-10^{\circ}$ of the center of gaze). Panels $b$ and $c$ show the corresponding neuronal responses to moving dots (black traces), moving gratings (gray traces) and static gratings (green traces). The black and green line segments below or above the activity traces denote the corresponding visual stimulation epochs for the moving (dots and gratings) and static (grating) stimuli, respectively. Panel c illustrates the averaged (10 trials) neuronal responses for the 12 directions of motion tested. The polargram in the middle represents the mean response rate for the 12 directions averaged over the stimulation epoch. The data points used to construct the polarplot for orientation (green trace) is based on 6 orientations. These 6 data points were mirrored in order to generated a 12-point polarplot, facilitating the comparison between orientation and direction selectivity. The circular dashed line at the center of the polar plot depicts the averaged spontaneous activity. Note that the response to moving gratings were characteristically weaker than those to moving dots (maximum average response of 119 spikes per second or $\mathrm{s} / \mathrm{s}$ ). However, the general selectivity profile was similar for both moving stimuli (optimal response at $60^{\circ}$ ). Static gratings elicited optimal responses at an orientation orthogonal (i.e., $150^{\circ}$ ) to the preferred axis of movement (compare the black/gray vs. green traces of the polargram and the two inserts to the left). Panels in B show enlarged versions of the histograms for the preferred direction of motion $\left(60^{\circ}\right.$, left panel) and for the preferred orientation axis $\left(150^{\circ}\right.$, right panel). Asterisks indicated the corresponding conditions in (b) and (c). 
a1

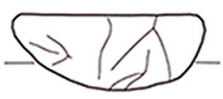

b1

$70 \mathrm{~s} / \mathrm{s}$

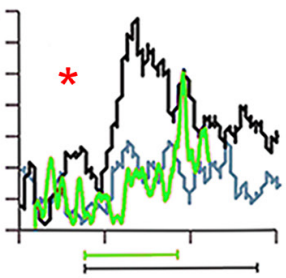

Type II Unidirectional

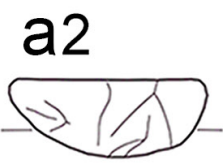

b2
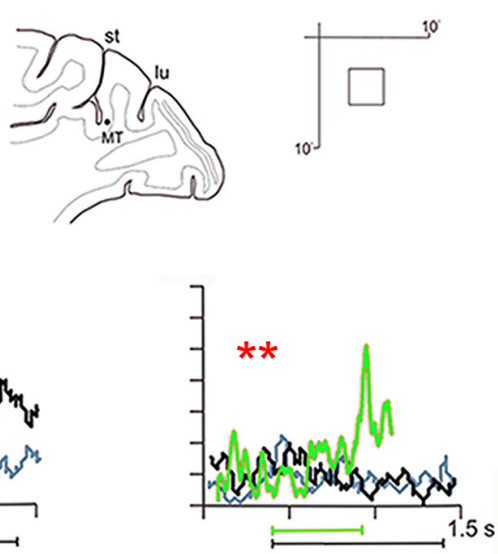
$1.5 \mathrm{~s}$

\section{Type II Unidirectional}

$60 \mathrm{~s} / \mathrm{s}$

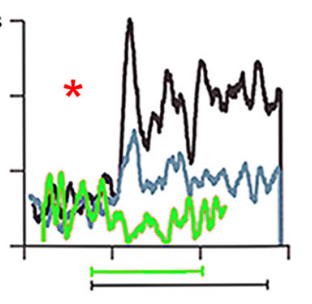

Type DS Unidirectional

a3
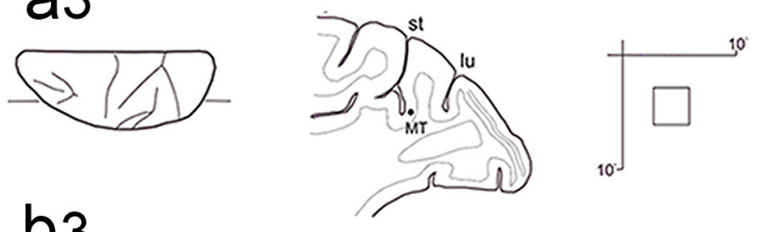

b3
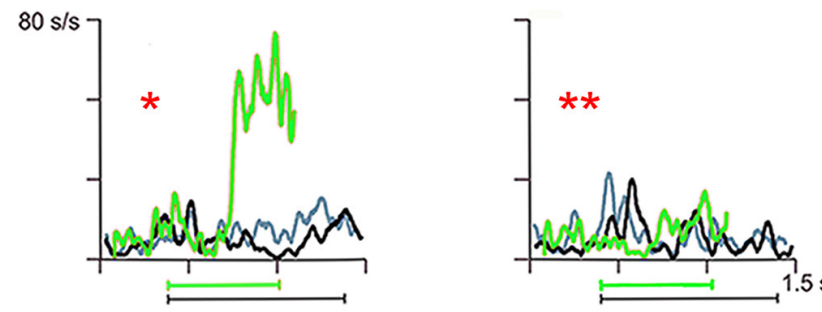
$1.5 \mathrm{~s}$

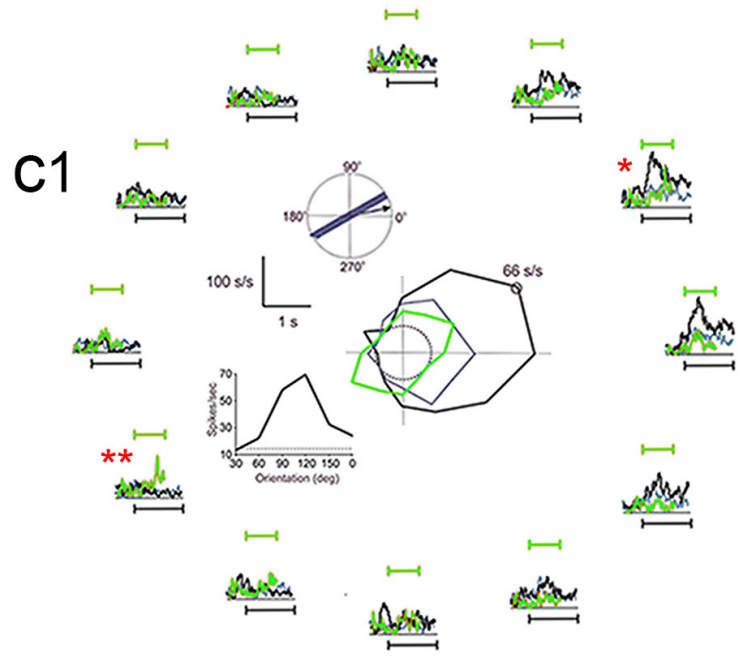

C2
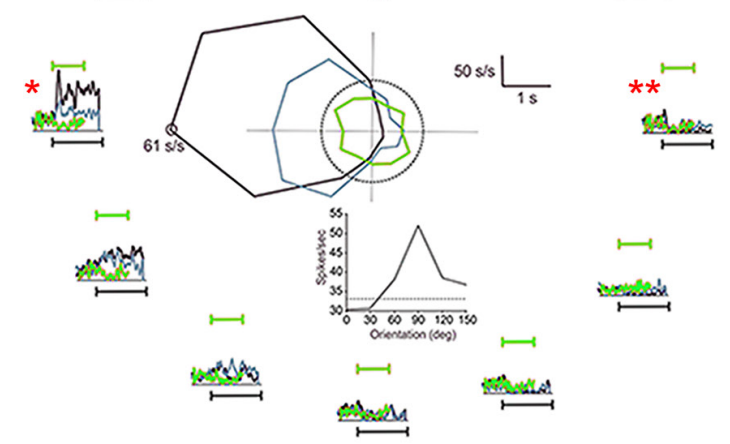

$\longmapsto$

$\stackrel{\text { insthods }}{\longmapsto}$

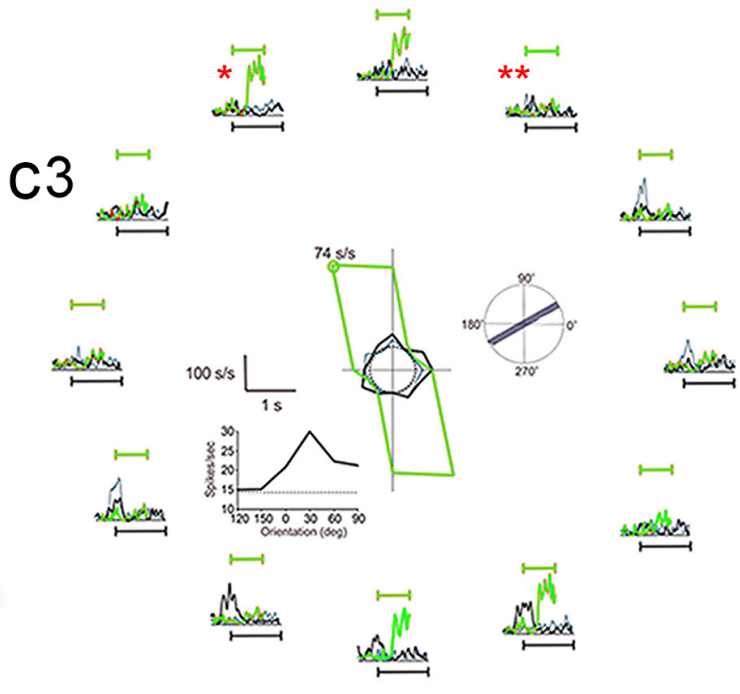

Figure 3. Examples of MT sites showing Type II unidirectional activity, Type DS unidirectional activity and Type OS activity (top, middle and bottom panels, respectively). Conventions as in Fig. 2. 
the responsive sites fired selectively to a single direction, and that most of the sites were selective to either one or two directions of motion. In order classify neurons as Type I or Type II cells, we computed the difference between the axis of motion and orientation selectivity across the population (Fig. 1C). This difference was required to be between 60 to 90 degrees for Type I cells, and smaller than 30 degrees for Type II cells. Type I unidirectional cells were more frequent than Type II unidirectional cells $(p<0.05)$, and non-oriented pan-directional cells were more frequent than pan-directional orientation selective cells $(p<0.01)$.

\begin{tabular}{|c|c|c|c|c|c|c|c|c|c|c|}
\hline & $\rightarrow$ & & & & $\rightarrow$ & & $\uparrow$ & $\leftrightarrow$ & $\pi$ & $\nearrow$ \\
\hline & * & $\searrow$ & $\leftrightarrow$ & $\hat{\imath}$ & $\lambda$ & $\nwarrow$ & $\uparrow$ & $\leftarrow$ & $\nearrow$ & $\uparrow$ \\
\hline T & $\leftrightarrow$ & $\lambda$ & $\nearrow$ & $\swarrow$ & 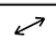 & $\downarrow$ & $\uparrow$ & $\leftrightarrow$ & $\rightarrow$ & $\uparrow$ \\
\hline * & * & & $\leftarrow$ & & & $\downarrow$ & $\pi$ & $\uparrow$ & $\rightarrow$ & $\uparrow \uparrow$ \\
\hline$\uparrow$ & * & $\uparrow$ & $\downarrow$ & * & $\leftrightarrow$ & $\downarrow$ & $\leftarrow$ & $\searrow$ & $\swarrow$ & $\rightarrow$ \\
\hline & * & * & $\leftarrow$ & 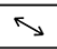 & $\rightarrow$ & $\leftarrow$ & $\rightarrow$ & $\rightarrow$ & $\searrow$ & $\downarrow$ \\
\hline & * & $\Omega^{\prime}$ & & $\lambda$ & * & $\kappa$ & $*$ & $\lambda$ & $\rightarrow$ & $\leftarrow$ \\
\hline$\downarrow$ & $\downarrow$ & $\leftrightarrow$ & * & $\uparrow$ & & $\leftrightarrow$ & $\checkmark$ & $\nearrow$ & $\uparrow$ & $\pi$ \\
\hline & $*$ & & $*$ & $\downarrow$ & & $\longleftrightarrow$ & $\leftrightarrow$ & $*$ & 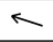 & 1 \\
\hline * & $\uparrow$ & $\pi$ & $\hat{\imath}$ & $\rightarrow$ & & $s$ & $\checkmark$ & $\downarrow$ & $\pi$ & $\leftarrow$ \\
\hline * & $\uparrow$ & $<$ & $\uparrow$ & $\uparrow$ & $\uparrow$ & & $\uparrow$ & $\leftrightarrow$ & $\leftrightarrow$ & $\leftarrow$ \\
\hline$\leftrightarrow$ & * & $*$ & $*$ & $\uparrow$ & $\kappa$ & & & $\leftrightarrow$ & & $\leftarrow$ \\
\hline
\end{tabular}

b

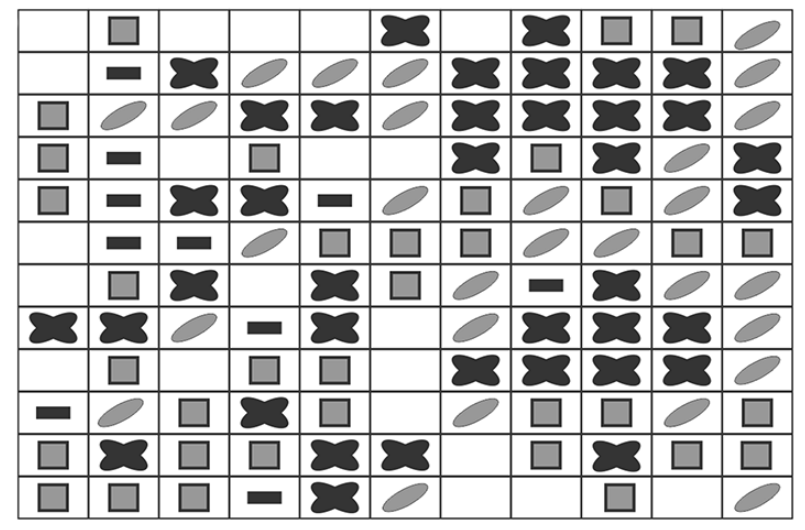

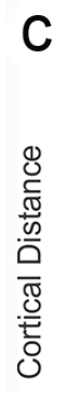
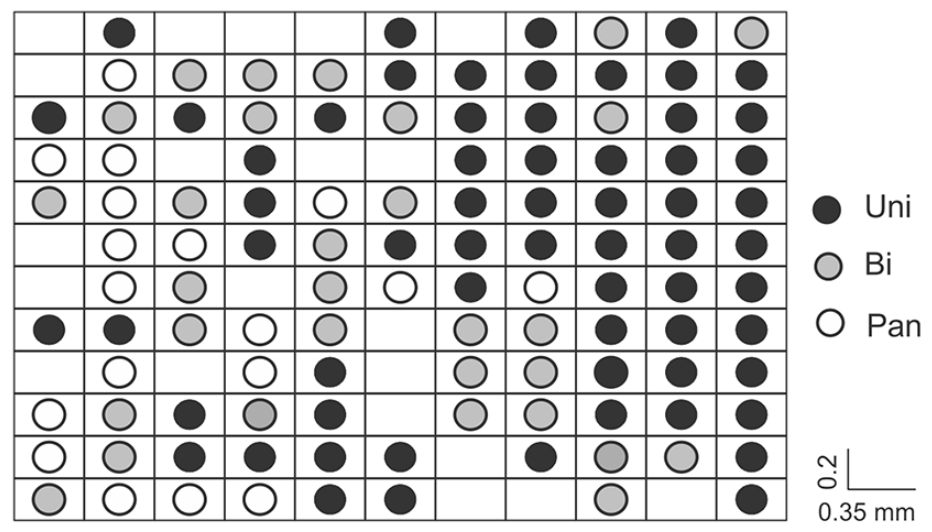

Cortical Distance
3 Type I

Type II

$\square$ TypeDS

- Typeos

Figure 4. Two-dimensional surface maps of area MT. (a) Selectivity profile for the tangential plane in which we obtained the largest number of responsive sites. (b) Two-dimensional surface map for cell type. (b) Twodimensional surface map for directions selectivity. 


\section{Type I cell}

Figure 2 exemplifies the response properties of a motion selective Type I unidirectional site (preferred direction of motion at $\sim 60$ degrees). Note that the tuning properties for this site are somewhat dependent on the type of stimulus used. It shows stronger responses and broader direction selectivity to moving dots (black traces) as compared to moving gratings (gray traces) with the same overall luminance. Note that the neuronal response is inhibited relative to spontaneous activity when dots and gratings are presented moving at the direction opposite (i.e., $\left.240^{\circ}\right)$ to the optimal. The presentation of static gratings at six possible orientations reveals that the orientation selectivity axis lies orthogonal to the direction selectivity axis. These properties characterize a Type I site.

Type II cell

The main characteristic of Type II neurons is to have similar selectivity axes for direction and orientation. The response properties of an example Type II unidirectional site (preferred direction of motion at $\sim 30$ degrees) is illustrated in Figure 3 (top panel). Note that there is no inhibition of neuronal activity relative to spontaneous activity when moving gratings and dots are shown at the direction opposite to the preferred (i.e., at approximately $210^{\circ}$, see polargram at $\mathrm{c} 1$ ). Additionally, note the off response to the 120 -degree static gratings (see the panels depicted with ** in b1 and $\mathrm{c} 1$ ). As we discuss later, this may be due to the release of early inhibition taking place at the circuit responsible for shaping orientation selectivity.

Type DS, non-oriented motion selective cell

Type DS cells are approximately as prevalent as Type I cells in area MT. The defining characteristic of Type DS cells is that they exhibit significant direction selectivity, but no selective response to stimulus orientation. As shown in Figure 3 (middle panel), this example site shows a strong selectivity to the $180^{\circ}$ direction of motion. As observed for the previous examples, moving dots elicit a stronger response than the one to moving gratings. However, both moving stimuli prompted marked inhibition of neuronal activity (relative to spontaneous activity) for the direction of movement opposite to the preferred $\left(\sim 0^{\circ}\right)$. As a matter of fact, neuronal activity is virtually silenced for dots moving at $0^{\circ}$. We found a subtle attenuation of the responses when static gratings were presented at all orientations. However, there was no difference in the responses for the different orientations tested. Note that we observed no significant response transient to stimulus onset or offset.

Type OS, orientation selective pan-directional cell

Type OS cells were the rarest category we observed in our sample of MT sites. Despite the fact that area MT is classically known for coding stimulus motion, this cell type shows no significant direction selectivity, but a clear selectivity for stimulus orientation. We are particularly interested in this cell type because it may be a key participant in the early hierarchy of MT circuits responsible for constructing direction selectivity. Figure 3 (bottom panel) shows an example of a Type OS site with strong neuronal responses to the $30^{\circ}$ oriented static grating. Response to moving gratings and dots were absent or very weak. Lack of responses to moving gratings was particularly intriguing. Despite the fact that a $120^{\circ}$ moving grating also exhibited a $30^{\circ}$ orientation, the simple fact that it moved was enough to hinder any neuronal response. As the Type DS site described above, 
this example of a Type OS site also did not show a significant transient response to stimulus onset or offset.

\section{Distribution of cell type and motion selectivity}

Figure 4 illustrates the tangential plane for which we obtained the largest number of responsive sites using our planar multielectrode array (see Diogo et al. 2003, for details). The corresponding plots reveal the two-dimensional distribution of cell type and directional selectivity in area MT, and thereby allows us to appreciate if any neighboring pattern among these classes emerges. Optimal direction selectivity was determined by the peak value obtained by fitting two gaussian function to the neuronal responses obtained for the 12 directions of stimulus motion (Fig. 4a). Figure 4b shows 38 Type I sites (black cross), 28 Type II sites (gray oval), 35 Type DS sites (gray square) and 10 Type OS sites (black bar). Unclassified sites are shown in white. In order to investigate our entire population of sites, we performed the next neighbor analysis for all the three recording planes in area MT. Type I sites had 76 Type I, 56 Type II, 47 Type DS, 16 Type OS and 29 unclassified sites as neighbors; Type II sites had 64 Type I, 44 Type II, 47 Type DS, 13 Type OS and 23 unclassified sites as neighbors; Type DS sites had 60 Type I, 46 Type II, 75 Type DS, 28 Type OS and 33 unclassified sites as neighbors. Finally, Type OS sites had 17 Type I, 11 Type II, 16 Type DS, 9 Type OS and 10 unclassified sites as neighbors. Therefore, Type I sites were not randomly scattered $\left(x^{2}=4.46, p=0.035\right)$, showing a tendency to cluster. The same is not true for Type II sites $\left(x^{2}=2.367, p=0.124\right)$. Inasmuch as Type I sites are more frequent, there was no difference in the type of neighbor for both Type I and Type II cells ( $\left.X^{2}=0.056, p=0.813\right)$.

The distribution of the direction selectivity profile is shown in Figure 4c. Here, we were able to classify 111 MT sites: 63 unidirectional sites (black circle), 30 bidirectional sites (gray circle) and 18 pan-directional sites (white circle). In order to investigate our entire population of sites, we performed the next neighbor analysis for all the three recording planes in area MT. This classification was done regardless of whether the site significantly responded to the static grating stimuli. Next neighbor analysis revealed: unidirectional sites had 274 unidirectional, 80 of bidirectional, and 38 pandirectional sites as neighbors; bidirectional sites had 73 unidirectional, 42 bi-directional and 37 pan-directional sites as neighbors. Finally, pan-directional sites had 39 unidirectional, 32 bidirectional, and 14 pan-directional sites as neighbors. Thus, the distribution of unidirectional sites was not randomly scattered $\left(x^{2}=3.4, p=0.03\right)$, showing a tendency to cluster with other unidirectional sites (e.g., see the right portion of Fig. 4c). On the other hand, bidirectional and pan-directional sites showed no preference for any particular neighbor.

\section{Relationship of cell type and direction selective classes}

Despite the fact that the distributions of Type I and unidirectional sites across MT surface were not random and exhibited significant clustering, the clustering patterns between these two classes were independent and showed no correlation with one another. A similar phenomenon can be observed in $\mathbf{V} 1$, for example, where two conspicuous organizational maps, namely orientation columns and ocular dominance columns coexist but are uncorrelated.

Figure 5a shows the next neighbor probability for each cell type and each motion selectivity based on the percentage of comparable neighbors for each site, at different distances in a circle of $500 \mu \mathrm{m}$-radius. Within a given cluster, the probability remains constant in a circle of approximately the size of the cluster. We also 

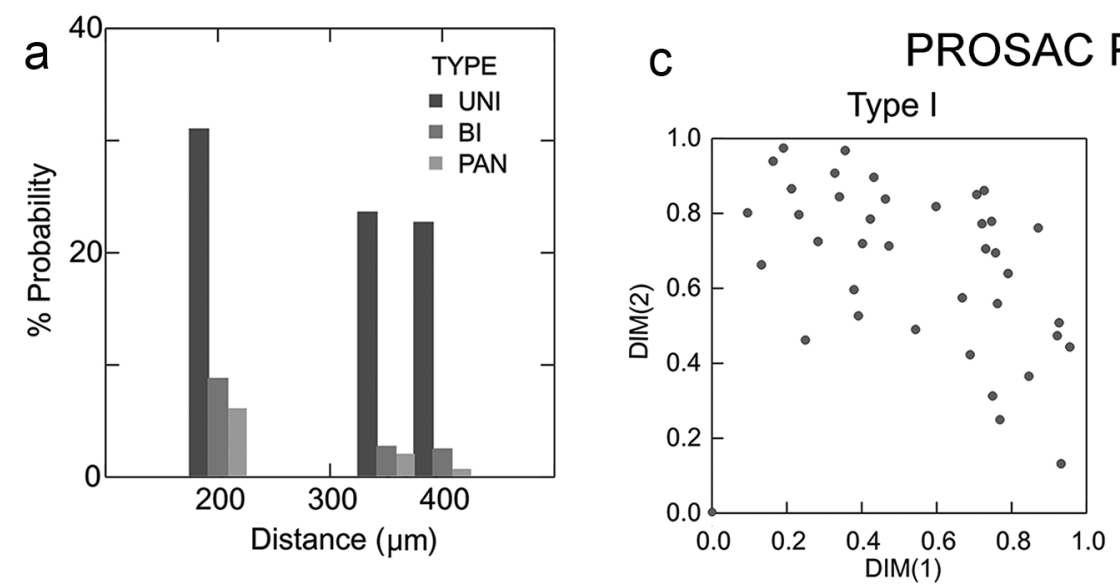

\section{Profile Plots}
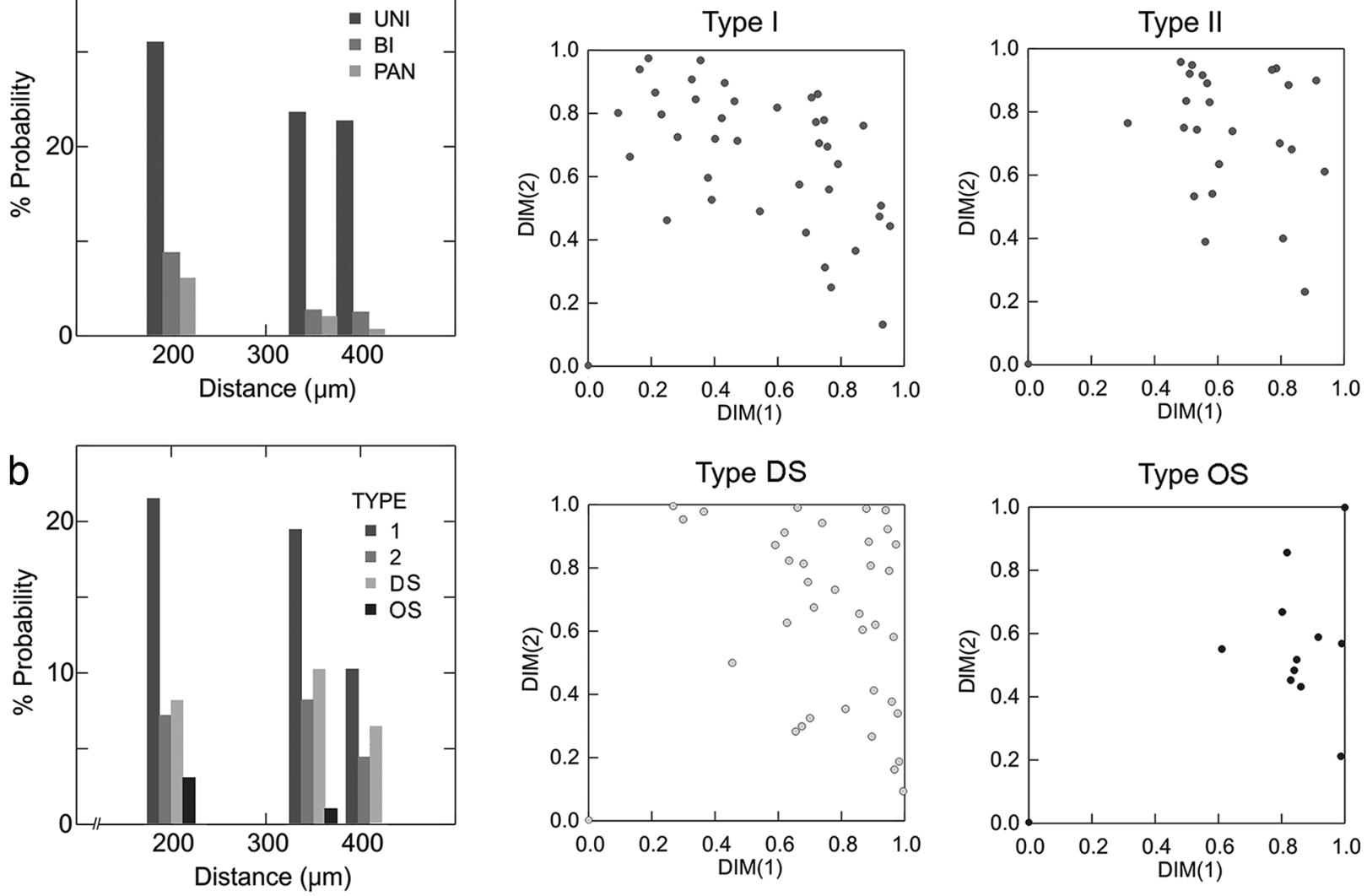

Figure 5. Clustering analysis of direction selectivity and cell type in the surface dimension of area MT. (a) Clustering of direction selectivity evaluated using next neighbor probability (PROSAC). The clustering probability is plotted as a function of distance on the surface dimension. Unidirectional sites showed significant clustering at around $200 \mu \mathrm{m}$ (note the decay > $300 \mu \mathrm{m}$ ). (b) Clustering of cell type evaluated using next neighbor probability (PROSAC). Type I cells were significantly clustered at around $300 \mu \mathrm{m}$ (note the marked decay on probability of occurrence $>400 \mu \mathrm{m}$ ). Type OS cells showed a trend for clustering at around $200 \mu \mathrm{m}$, but the result did not reach statistical significance. (c) PROSAC profile plots for each of the four cell types.

used the half-width at half-height distance of the first (fundamental) component of the 2D-FFT power spectrum to estimate the dimensions of the unidirectional $(>500 \mu \mathrm{m})$ and bi-directional $(400 \mu \mathrm{m})$ clusters, as well as the Type I $(400 \mu \mathrm{m})$, Type $I I(300 \mu \mathrm{m})$, and type DS $(<300 \mu \mathrm{m})$ clusters. Therefore, the size of the cluster was estimated based on both the cutoff of the first component, as well as by the distance at which the next neighbor probability falls abruptly. Figure $5 c$ shows the PROSAC profile plots of partially ordered scalograms of the XY-coordinates of the location for the entire population (Figure 5a) and for each class of site (Figure 5b) to evaluate the cluster arrangement based on the histograms of the magnitudes of the vectors (Figure $5 \mathrm{c}$ ) of each interaction among similar sites (Z1-Zn distances). Those plots suggest that each group has a unique but overlapping size of clusters.

\section{DISCUSSION}

Area MT in the capuchin monkey (a New World monkey) is homologous to area MT of both Old World monkeys (Fiorani et al. 1989, Gattass \& Gross, 1981) and humans (Tootell \& Taylor 1995). 
It is considered one of the first stages of motion processing. The activity of MT neurons has a direct correlate with perception. For example, manipulating the coherence of random dot patterns directly correlates with the firing rate of individual MT neurons, and bears a direct relationship with the psychometric function for motion perception (Newsome \& Stein-Aviles 1999).

MT is an oval area mainly located in the posterior bank of the dorsal portion of the superior temporal sulcus (STS). In order to describe the distribution of cell types and direction selectivity in the two-dimensional surface of area MT, we used a planar multielectrode array that recorded multiunit responses at an angle tangential to its cortical layers. The majority (85\%) of the visually responsive sites were selective to one or two directions of motion (i.e., direction selective sites), while the remaining $15 \%$ were pandirectional. About $57 \%$ of the sites were selective to orientation. We classified our sites as Type I, II, DS or OS depending on their selectivity for a
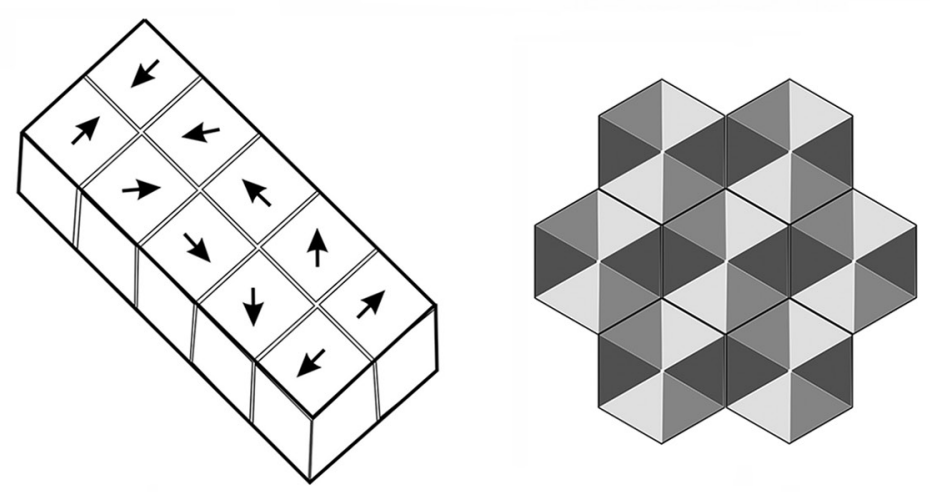
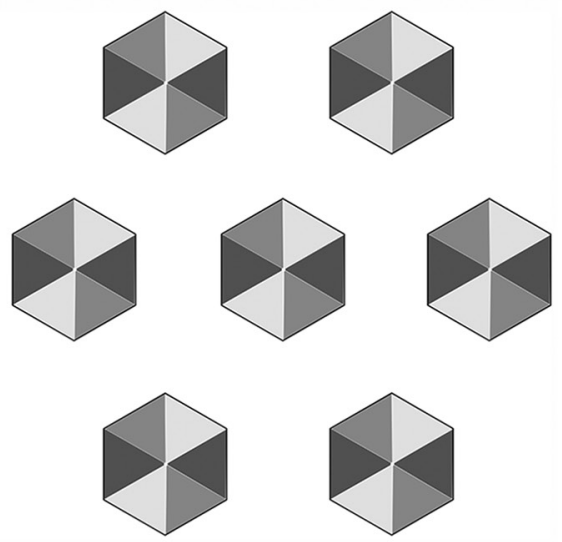

\section{$\mathrm{b}$}
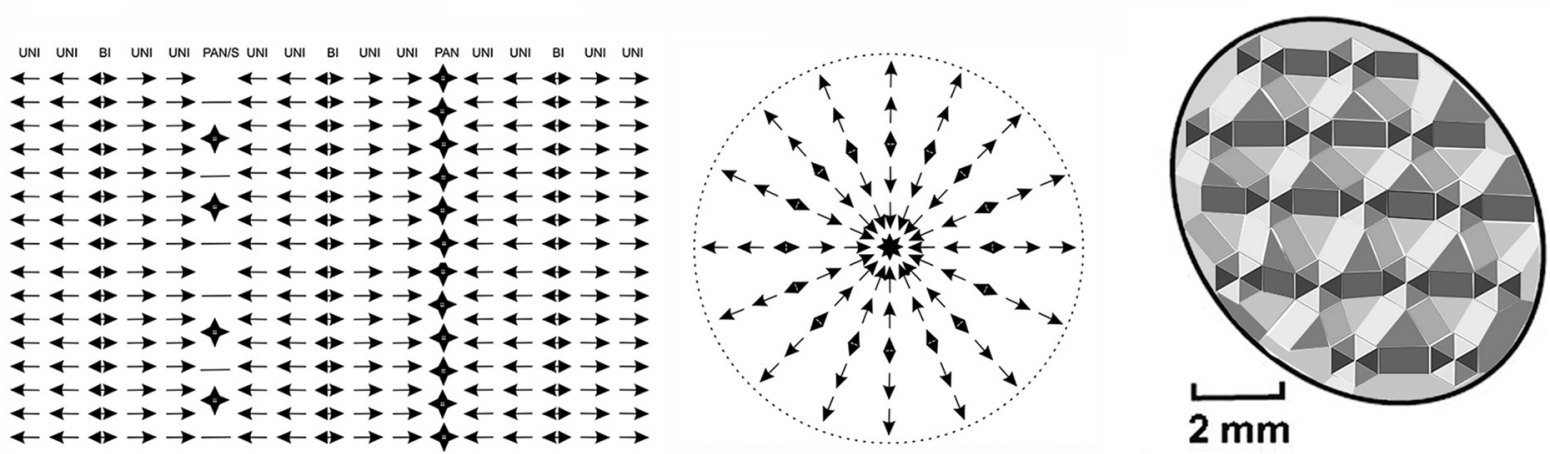

Figure 6. A model for the combined arrangement of cell type and directional selectivity in the surface dimension of area MT. (a) Left: The ice-cube model proposed by Albright et al (1984), where columns with opposing directional selectivity are organized into larger modules coding for axis of motion. This model assumes a smooth transition of direction selectivity across MT surface, with occasional 180-degree flips. Middle: Subsequent work by Diogo et al (2003) suggests that smooth transitions in direction selectivity coexist with singularity points (i.e., pinwheels). The pinwheels are actually more sparsely distributed across MT surface than initially expected, with relatively large intervening slabs coding for the same direction (right). Different shades of gray represent regions with different axis of movement selectivity. (b) Left: a modified model relative to the one illustrated in (a), where bidirectional and pan-directional modules are incorporated. Middle: A model that incorporates pinwheels and direction selectivity columns. Right: A unified model that incorporates pinwheels, direction selectivity columns and elongated regions on MT surface coding for axis of motion. 
motion direction or stimulus orientation. We employed criteria similar to the ones used by Albright et al. (1984) and later by Rodman et al. (1989) to classify MT neurons as Type I or Type II cells. In capuchins, as in macaques, there is a predominance of Type I cells. Moreover, Type I and Type II cells are predominantly unidirectional, and the majority of bi-directional sites are Type I. Finally, we observed that Type I sites had a significant tendency to cluster together, and that unidirectional sites also had a significant tendency to cluster together. However, cell type and directional selectivity showed no correlation with each other in their surface distribution across area MT.

\section{Principles of Cortical Organization}

Area MT of macaques cannot be readily evaluated by optical imaging, inasmuch as it is buried inside a deep STS sulcus. However, experimental evidence hinted that the owl monkey, which has a relatively flat cortex that is optically accessible, possesses a system of cortical columns in MT coding for stimulus direction (Baker et al. 1981). This result was later confirmed by Malonek et al. (1994) using optical imaging of intrinsic neuronal signals. The data showed a clear tendency for radial organization, similar to the orientation column pinwheels described in area V1. Using planar multielectrode arrays, we showed a combined pattern of linear and circular arrangements (Diogo et al. 2003).

Area MT is distinct relative to other cortical areas in that it shows a coarse visuotopic organization associated with a heavy myelination pattern. Additionally, metabolic enzymatic staining techniques reveal no clear repetitive distribution of cortical modules such as those observed, for example, in areas V1 and V2 (Gattass \& Gross 1981, Van Essen et al. 1981, Desimone \& Ungerleider 1986, Tootell et al. 1998). Albright et al. (1984) used electrode penetrations at different angles relative to the cortical surface to study the functional architecture of area MT. Based on the observation that direction selectivity occasionally reversed in 1800 , the authors proposed a columnar organization based on regular ice-cube modules (Fig. 6a, left). Later, Diogo et al. (2002, 2003) showed that this functional architecture in MT, based on linear gradual changes in direction selectivity, was integrated with a pattern of radially organized pinwheels. The arrangement was dominated by rectangular zones of directional selectivity, with scattered pinwheels along the surface (Fig 6a, center and right). The arrangement of unidirectional, bidirectional and pan-directional cells can be represented in the scheme shown in Figure $6 b$ (left) for the case of quasi-rectangular directional zones, and in the scheme shown in Figure 6b (middle) in the case of pinwheels. We estimated MT surface area in capuchins to be about $90 \mathrm{~mm}^{2}$ (Fiorani et al. 1989), and to contain 13-15 pinwheels arrangements and 18-22 polygonal patches of direction selectivity (Diogo et al. 2002, 2003).

\section{Independent maps for cell type and direction selectivity}

During ontogenesis, the establishment of visual topography in V1 is followed by the appearance of ocular dominance columns, which is then followed by the appearance of orientation columns (Hubel \& Wiesel 1974, Levay et al. 1975). Following this plan, some features such as visual topography are established first and are then followed by the development of other compartmental organizations. It is thereby possible to hypothesize that MT follows a similar scheme. First, visual topography is established. Second, circuits are constructed to extract specific properties of the visual stimulus, giving rise to unidirectional, bidirectional and 
pan-directional neurons, as well as to different cell types.

\section{Perceptual correlates of cell type}

Work by Movshon et al. (1985) used superimposed sinusoidal gratings (i.e., plaids) to show that one specific population of MT neurons (Type I cells) responded to the individual components of the stimulus, while another population (Type II cells) responded to the combined (i.e., pattern) motion of the plaids. Notably, the two cell types were directly correlated with the perception of component or pattern motion (Movshon et al. 1985, Stoner \& Albright 1998). Subsequent work by Wang \& Movshon (2016) reported that MT cells with higher index for pattern motion correspond to Type II cells. Additionally, these neurons tended to have stronger direction selectivity, broader direction tuning to gratings, better response to plaids compared to gratings, smaller receptive fields and stronger surround suppression. Based on these findings, the model proposed by Rust et al. (2006) suggests that pattern responses emerge from the pooling of V1 inputs with a wide range of spatial-temporal tuning.

\section{Areas downstream to area MT}

The dorsal stream of visual information processing includes area MT, the MST complex and part of Area 7 in the parietal lobe. The medial superior temporal area (MST) was originally defined by the limits of the MT projections zone located in the dorsal and medial portions of STS, adjacent to MT (Van Essen et al. 1981, Maunsell \& Van Essen 1983, Ungerleider \& Desimone 1986) and lateral to TPOC (anterior STS bank) (Hikosaka et al. 1988, Lewis \& Van Essen 2000a, b). This region, named the MST complex by Lewis \& Van Essen (2000a), is involved in processing optic flow information (often due to the movement of the observer), and may be related to the processing of object visual motion (Eifuku \& Wurtz, 1998, 1999). Distinct zones in the MST complex were functionally defined by the properties of their neurons. They responded exclusively to visual stimuli, had receptive fields that were larger than those found in MT, most were direction selective, and exhibited only a crude topographic organization of the visual field (Van Essen et al. 1981, Saito et al. 1986, Desimone \& Ungerleider, 1986, Fiorani et al. 1989).

Based on the distinct response characteristics of its neurons, Saito et al. (1986) subdivided the MST complex into two functional sub-regions: one more medial and dorsal where direction size-rotation-cells are found, and another more lateral sub-region with few direction selective neurons. These data suggest that the dorsolateral stream of visual information processing, as described by Nascimento-Silva et al. (2003), can be hierarchically organized. In the context where cells or discrete circuits in one specific area exhibit different response properties corresponding to the different stages of neuronal processing, we hypothesize that Type I and Type II cells in MT participate as integral components responsible for building up this network. Type OS cells, on the other hand, could be projecting to more complex networks at higher areas, consistent with a more symbolic or abstract level of neuronal processing.

\section{Acknowledgments}

We are grateful to Elaine S. Alves for her precious help with data analysis. We also wish to thank Edil Saturato da Silva Filho, Liliane Herringer Motta and Thereza Monteiro for skillful technical assistance. This study was supported by PRONEX, Fundação de Amparo à Pesquisa do Estado do Rio de Janeiro (FAPERJ E-26/010.001.238/2016, E-26/110.905/2013), Serrapilheira Institute (grant\# Serra-1709-17523), Conselho Nacional de Desenvolvimento Científico e Tecnológico (CNPq, 471.166/2013-8) and Financiadora de Estudos e Projetos (FINEP, 0354/16). We declare no conflict of interest. 


\section{REFERENCES}

ALBRIGHT TD. 1984. Direction and orientation selectivity of neurons in visual area MT of the macaque. J Neurophysiol 52: $1106-1130$.

ALBRIGHT TD, DESIMONE R \& GROSS CG. 1984. Columnar organization of directionally selective cells in visual area MT of the macaque. J Neurophysiol 51: 16-31.

BAKER JF, PETERSEN SE, NEWSOME WT \& ALLMAN JM. 1981. Visual response properties of neurons in four extrastriate visual areas of the owl monkey (Aotus trivirgatus): A quantitative comparison of medial, dorsomedial, dorsolateral and middle temporal areas. J Neurophysiol 45: 397-416.

BARLOW HB, BLACKMORE C \& PETTIGREW JD. 1967. The neural mechanism of binocular depth discrimination. J Physiol 193: 327-342.

BARLOW HB. 1986. Why have multiple cortical areas? Vision Research 51: 16-31.

CHUM O \& MATAS J. 2010. Large-scale discovery of spatially related images. IEEE Trans Pattern Anal Mach Intell 32: 371-377.

DESIMONE R \& UNGERLEIDER L. 1986. Multiple visual areas in the caudal superior temporal sulcus of the macaque. J Comp Neurol 248: 164-189.

DIOGO ACM, SOARES JGM, ALBRIGHT TD \& GATTASS R. 1999. Columnar organization of area MT in Cebus apella. Soc Neurosci Abs 25: 672.

DIOGO ACM, SOARES JGM, ALBRIGHT TD \& GATTASS R. 2002. TwOdimensional map of directional selectivity in cortical visual area MT of Cebus monkey. An Acad Bras Cienc 74: 463-473.

DIOGO ACM, SOARES JGM, KOULAKOV A, ALBRIGHT TD \& GATTASS R. 2003. Electrophysiological imaging of functional architecture in cortical visual area MT of Cebus monkey. J Neurosci 23: 3881-3898.

EIFUKU S \& WURTZ RH. 1998. Response to motion in extrastriate area MSTI: center-surround interactions. The American Physiological Society 282-296.

EIFUKU S \& WURTZ RH. 1999. Response to motion in extrastriate area MSTI: disparity sensitivity. The American Physiological Society 2462-2475.

FIORANI JR M, GATTASS R, ROSA MG \& SOUSA AP. 1989. Visual area $M T$ in the Cebus monkey: location, visuotopic organization, and variability. J Comp Neurol 287: 98-118.

GALLYAS F. 1979. Silver staining of myelin by means of physical development. Neurol Res 1: 2039.
GATTASS R \& GROSS CG. 1981. Visual topography of the striate projection zone (MT) in posterior superior temporal sulcus of the macaque. J Neurophysiol 46: 621-638.

GATTASS R, SOUZA APB \& COVEY E. 1985. Cortical visual areas of the macaque: possible substrates for pattern recognition mechanisms. In: Chagas C, Gattass R \& Gross CG (Eds), Study Week on Pattern Recognition Mechanisms. Pontificiae Academiae Scientiarum Scripta Varia 54: 1-20.

GATTASS R, SOUZA APB \& GROSS CG. 1988. Topographic organization and extent of $\mathrm{V} 3$ and $\mathrm{V} 4$ of the macaque. J Comp Neurol 8: 1831-1845.

GATTASS R ET AL. 2005. Cortical visual áreas in monkeys: location, topography, connections, columns, plasticity and cortical dynamics. Philos Trans R Soc Lond B Biol Sci 360: 709-731.

GROSS CG, ROCHA-MIRANDA CE \& BENDER DB. 1972. Visual properties of neurons in inferotemporal cortex of the macaque. J. Neurophysiol 35: 96-111.

GROSS CG, BRUCE CJ, DESIMONE R, FLEMING J, \& GATTASS R. 1981. Cortical visual areas of the temporal lobe: Three areas in the macaque, In: Woolsey CN (Ed), Cortical Sensory Organization Volume 2: Multiple Visual Areas. Humana Press, Clifton, NJ, p. 187-216.

HIKOSAKA K, IWAI E, SAITO HA \& TANAKA K. 1988. Polisensory properties of neurons in the anterior bank of the caudal superior temporal sulcus of the Macaque monkey. J Neurophysiol 60: 1615-1637.

HUBEL DH \& WIESEL TN. 1962. Receptive fields, binocular interaction and functional architecture in the cat's visual cortex. J Physiol 160: 106-154.

HUBEL DH \& WIESEL TN. 1968. Receptive fields and functional architecture of monkey striate cortex. J Physiol 195: 215-243.

HUBEL DH \& WIESEL TN. 1974. Sequence regularity and geometry of orientation columns in the monkey striate cortex. J Comp Neurol 158: 267-294.

KAAS JH. 1989. Why does the brain have so many visual areas? J Cogn Neurosci 1: 121-135.

LEVAY S, HUBEL DH \& WIESEL TN. 1975. The pattern of ocular dominance columns in macaque visual cortex revealed by a reduced silver stain. J Comp Neurol 159: 559-576.

LEWIS JW \& VAN ESSEN DC. 2000a. Mapping of architectonic subdivisions in the macaque monkey, with emphasis on parieto-occipital cortex. J Comp Neurol 428: 79-111. 
LEWIS JW \& VAN ESSEN DC. 2000b. Corticocortical connections of visual sensorimotor, and multimodal processing areas in the parietal lobe of the macaque monkey. J Comp Neurol 428: 112-137.

MARTIN KAC \& WHITTERIDGE D. 1984. Form, function and intracortical projections of spiny neurons in the striate visual cortex of the cat. J Physiol (London) 353: 463-504.

MAUNSELL JH \& VAN ESSEN DC. 1983. The connections of the middle temporal visual area MT and their relationship to a cortical hierarchy in the macaque monkey. J Neurosci 3: 2563-2586.

MALONEK D, TOOTELL RB \& GRINVALD A. 1994. Optical imaging reveals the functional architecture of neurons processing shape and motion in owl monkey area MT. Proc R Soc Lond B Biol Sci 258: 109-119.

MOVSHON JA, ADELSON EH, GIZZI MS \& NEWSOME WT. 1985. The analysis of moving visual patterns. In: Chagas C, Gattass R \& Gross C (Eds), Study Week on: Pattern Recognition Mechanisms. Vatican City: Pontificia Academia Scientiarum 54: 117-151.

NASCIMENTO-SILVA S, GATTASS R, FIORANI M JR \& SOUSA AP. 2003. Three streams of visual information processing in V2 of Cebus monkey. J Comp Neurol 466: 104-118.

NEUENSCHWANDER S, GATTASS R, SOUSA APB \& PIÑON MCG. 1994. Identification and visuotopic organization of areas PO and POd in Cebus monkey. J Comp Neurol 340: 65-86.

NEWSOME WT \& WURTZ RH. 1981. Response properties of single neurons in the middle temporal visual (MT) of alert macaque monkey. Soc Neurosci Abst 7: 832.

NEWSOME WT, MIKAMI A \& WURTZ RH. 1986. Motion selectivity in macaque visual cortex. III. Psychophysics and physiology of apparent motion. J Neurophysiol 55: 1340-1351.

NEWSOME WT \& STEIN-AVILES JA. 1999. Nonhuman Primate Models of Visually Based Cognition. ILAR J 39: 78-91.

RODMAN HR, GROSS CG \& ALBRIGHT TD. 1989. Afferent basis of visual response properties in area MT of the macaque. I. Effects of striate cortex removal. J Neurosci 9: 2033-2050.

RODMAN HR, GROSS CG \& ALBRIGHT TD. 1990. Afferent basis of visual response properties in area MT of the macaque. II. Effects of superior colliculus removal. J Neurosci 10: $1154-1164$.

RUST NC, MANTE V, SIMONCELLI EP \& MOVSHON JA. 2006. HOW MT cells analyze the motion of visual patterns. Nat Neurosci 9: 1421-1431.

SAITO H, YUKIE M, TANAKA K, HIKOSAKA K, FUKADA Y \& IWAI E. 1986. Integration of direction signals of image motion in the superior temporal sulcus of the macaque monkey. J Neurosci 6: 145-157.

STONER GR \& ALBRIGHT TD. 1998. Luminance contrast affects motion coherency in plaid patterns by acting as a depth-from-occlusion cue. Vision Res 38: 387-401.

TANAKA M, WEBER H \& CREUTZFIELD OD. 1986. Visual properties and spatial distribution of neurons in the visual association area on the prelunate gyrus of the awake monkey. Exp Brain Res 65: 11-37.

TOOTELL RBH, \& TAYLOR JB.1995. Anatomical evidence for MT and additional cortical areas in humans. Cereb Cortex 5: 39-55.

TOOTELL RB, HADJIKHANI N, HALL EK, MARRET S, VADUFFEL W, VAUGHAN JT \& DALE AM. 1998. The retinotopy of visual spatial attention. Neuron 6: 1409-1422.

UNGERLEIDER LG \& MISHKIN M. 1979. The striate projection zone in the superior temporal sulcus of macaca mulatta: location and topographic organization. J Comp Neurol 188: 347-366.

UNGERLEIDER LG \& MISHKIN M. 1982. Two cortical visual systems. In: Ingle DI, Goodale MA \& Mansfield RIW (Eds), Analysis of visual behavior, Cambridge MA, MIT Press, $p$. 549-586.

UNGERLEIDER LG \& DESIMONE R. 1986. Cortical connections of visual area MT in the macaque. J Comp Neurol 248: 190-222.

VAN ESSEN DC, MAUNSELL JHR \& BIXBY JL. 1981. The middle temporal visual area in the macaque: myeloarchitecture, connections, functional properties and topographic connections. J Comp Neurol 199: 293-326.

VAN ESSEN DC \& ZEKI SM. 1978. The topographic organization of rhesus monkey prestriate cortex. J Physiol (Lond) 277: 193-226.

WANG HX \& MOVSHON JA. 2016. Properties of pattern and component direction-selective cells in area MT of the macaque. J Neurophysiol 115: 2705-2720.

WURTZ RH, YAMASAKI DS, DUFFY CJ \& ROY JP. 1990. Functional specialization for visual motion processing in primate cerebral cortex. Cold Spring Harbor Symp Quant Biol 55: 717-727.

ZEKI SM. 1969. Representation of central visual fields in prestriate cortex of monkey. Brain Res 14: 271-291.

ZEKI SM. 1974. Functional organization of a visual area in the posterior bank of the superior temporal sulcus of the rhesus monkey. J Physiol 236: 549-573. 
ZEKI SM. 1983a. Colour coding in the cerebral cortex: the reaction of cells in monkey visual cortex to wavelengths and colours. Neuroscience 9: 741-765.

ZEKI SM. 1983b. Colour-coding in the cerebral cortex: The responses of wavelength-selective and colour-coded cells in monkey visual cortex to changes in wavelength composition. Neuroscience 9: 767-781.

\section{How to cite}

DIOGO ACM, LIMA B, SOARES JGM \& GATTASS R. 2020. Tangential distribution of cell type and direction selectivity in monkey area MT. An Acad Bras Cienc 92: e20190564. DOI 10.1590/0001-3765202020190564.

Manuscript received on May 16, 2019;

accepted for publication on October 19, 2019

\section{ANTONIA CINIRA M. DIOGO}

https://orcid.org/0000-0002-8616-6575

\section{BRUSS LIMA}

http://orcid.org/0000-0001-6865-2900

\section{JULIANA G. M. SOARES}

http://orcid.org/0000-0002-0408-8607

\section{RICARDO GATTASS}

http://orcid.org/0000-0002-0321-1490

Universidade Federal do Rio de Janeiro, Instituto de Biofísica Carlos Chagas Filho, Av. Carlos Chagas Filho 373, Bloco G,

Cidade Universitária, 21941-900 Rio de Janeiro, RJ, Brazil

Correspondence to: Ricardo Gattass

E-mail:rgattass@gmail.com

\section{Author contributions}

A.C.M.D, R.G. designed research; A.C.M.D, J.G.M.S., B.L., R.G. performed research; A.C.M.D, R.G. analyzed data; R.G. prepared the figures; R.G., B.L., J.G.M.S. wrote the paper.

\section{(cc) BY}

